\title{
A Hybrid Framework for Heart Disease Prediction Using Machine Learning Algorithms
}

\author{
L.Chandrika $^{1, *}$ and K.Madhavi ${ }^{2}$ \\ ${ }^{1}$ PG Student, Computer Science and Engineering, GRIET, Hyderabad, Telangana, India. \\ ${ }^{2}$ Professor, Computer Science and Engineering, GRIET, Hyderabad, Telangana,India.
}

\begin{abstract}
Cardiovascular Diseases (CVDs) are the primary cause for the sudden death in the world today from the past few years the disease has emerged greatly as a most unpredictable problem, not only in India the whole planet facing the criticality. So, there is a desperate need of valid, accurate and practical solution or application to diagnose the CVD problems in time for mandatory treatment. Predicting the CVD is a great challenge in the health care domain of clinical data analysis. Machine learning Algorithms (MLA) and Techniques has been vastly developed and proven to be effective and efficient in predicting the problems using the past data. Using these MLA techniques and taking the clinical dataset which provided by the healthcare industry. Different studies were takes place and tried only a small part into predicting CVD with ML Algorithms. In this thesis, we propose the different novel methodology which concentrates at finding appropriate features by using MLA techniques resulting at finding out the accurate model to predict CVD. In this prediction model we are trying to implement the models with different combinations of features and several known classification techniques such as Deep Learning, Random Forest, Generalised Linear Model, Naïve Bayes, Logistic Regression, Decision Tree, Gradient Boosted trees, Support Vector Machine, Vote and HRFLM and we have got an higher accuracy level and of $75.8 \%, 85.1 \%, 82.9 \%, 87.4 \%, 85 \%, 86.1 \%$, $78.3 \%, 86.1 \%, 87.41 \%$, and $88.4 \%$ through the prediction model for heart disease with the hybrid random forest with a linear model (HRFLM).
\end{abstract}

\section{Introduction}

It is hard to recognize Cardio vascular Diseases (CVD) because of a couple of contributory risk factors like raised cholesterol levels, diabetes, hypertension, dropping heart rate etc., like different problems. Various methods like Neural Networks(NN) and Data Mining(DM) techniques are used to get to know the intensity of CVD among different patients. The intensity of the problem is arranged depends on diverse strategies like Naive Bayes(NB), K-NN, Decision Tree(DT), and Genetic Algorithm(GA) [1]. The idea of CVD is unpredictable also, hence, the contamination ought to be dealt with circumspectly. Not doing as such may impact the heart or cause abrupt passing. The perspective of clinical science strategies and information digging are used for finding various types of metabolic issues. DM with arrangement has an immense impact in the conjecture of CVD and information assessment. We also observe DT be used to predict the accuracy of situations relevant to CVD [2].

Different strategies have been utilized for information deliberation by utilizing realized information digging techniques for anticipating CVD. In this work, various readings have been completed to create an expectation model utilizing particular procedures and relating at least two strategies. The combination of new procedures ordinarily known as half breed strategies [3]. We present neural networks utilizing pulse time arrangement. This strategy utilizes different clinical records for expectation, for example, Right pack branch block (RPBB), Left group branch block(LGBB), AFIB, NSR, SBR, AFL, $\mathrm{PVC}$, and SDB to discover the specific state of the heart patient with CVD. The dataset with a spiral premise work organization RBFN is utilized for arrangement. The data further bifurcated into two parts as $70.0 \%$ of the data is considered for testing purpose and the rest of the $30 \%$ is considered for training purposes [4].

We additionally present a CADSS in the medication and examination field. In past work, the utilization of information mining strategies in the medical care departments has been appeared to set aside less effort for the forecast of illness with more precise outcomes [5]. We bring up the proposal which analyse the CVD utilizing the GA. This technique utilizes compelling affiliation rules deduced with the GA for competition choice, hybrid, and change which brings about the new proposed wellness work. For exploratory approval, we utilize the most popular dataset collected by UCI (Cleveland Dataset). We like to experiment and

*Corresponding author: lingalachandrika97@gmail.com 
see how outcomes end up being unmistakable when contrasted with a portion of by using supervised learning procedures. The most remarkable transformative calculation PSO is presented and a few standards are produced for CVD. The standards have been applied haphazardly with encoding methods which bring about the progress of the exactness generally speaking [6]. CVD is anticipated dependent on indications to be specific like sex, rate of heart, age, and numerous features. The ML algorithms combination of $\mathrm{NN}$ is presented, whose outcomes are more exact and dependable.

$\mathrm{NN}$ are usually considered the best apparatus for the prediction like Cardiovascular and brain tumour diseases. The proposed technique we are taking 13 features of CVD forecast. The outcomes show an improved degree of execution contrasted with the current techniques. The CAS has likewise become a predominant therapy mode in clinical industry in this decade. The CAS remind the event of major antagonistic cardiovascular occasions (MACE) of CVD patients that are older. Their assessment turns out to be vital. We create results using an ANN algorithm, which delivers great execution in the forecast of CVD. Neural organization strategies are presented, which join back probabilities as well as anticipated qualities from different archetype procedures. This model accomplishes an exactness level of up to $89.01 \%$ which is a solid outcome contrasted with past research. For all trials, the UCI's Cleveland CVD dataset is utilized with NN algorithm to enhance the presentation of CVD in the papers [7] and [8].

We have additionally seen latest improvements in AI, ML strategies utilized for the IoT too [9]. Machine Learning strategies on online data appeared to provide a precise recognizable evidence of IoT devices which are related with a network. The researcher Meidan et al. took a data and marked as IoT online data from nine individual cell phones, Personal Computers, and IoT gadgets. Utilizing Supervised learning methods, to make a hybrid meta classifier [10]. In initial also crucial stage, the classifier can recognize traffic created by non-IoT and IoT gadgets. In the subsequent level, every IoT gadget is related to a particular IoT gadget class [10]. DL is a best strategy for getting précised data from all sensors of IoT gadgets conveyed in difficult conditions [11]. Based on its multi-layer-structure, DL is also a most suitable to the IoT edge computing [12].

In this research, we present a strategy which call's the main aspect of this experiment is to enhance the performance accuracy of CVD detection. Numerous examinations have been led that outcomes in limitations of highlight choice for algorithmic use. Interestingly, the HRFLM technique utilizes all highlights with no limitations of highlight determination. Here we direct analyses used to distinguish the highlights of an AI calculation with a crossover technique. The examination results indicates that our proposed method has more grounded capacity to anticipate CVD contrasted with existing strategies.
Further, the research of this paper is coordinated as follows: Segment II explains about past CVD detection experiments, existing strategies, and methods accessible. Section III, explains the overview of methodology. The process of -'HRFLM' classification modelling, preprocessing the data, performance measure and feature selection, classification and algorithms is discussed in segment -IV. In segment $\mathrm{V}$ discussed regarding the dataset and experiment interface and its evaluation. In segment-VI discussed the assessment of different modelling techniques and their results. Segment VII concludes the experimentation and a few details on a future research

\section{Related Work}

There are abundant previous researches in these fields mentioned in this research paper. ANN been acquainted to generate the great precision forecast in the healthcare research field [6]. The backpropagations of Multi-layer-Perceptron (MLP) and ANN is used for prediction of CVD. The achieved outcomes compared with the outcomes of already researched models on a same environment and inferred the model should be improved [13]. The CVD dataset of different cases collected by the UCI is used to find patterns using NN, SVM, DT, and NB. The experimental results are used to differentiate the overall performance of each model and accuracies of these algorithmic models. The proposed strategy(Hybrid model) returns outcome of $86.82 \%$ while working on the F-measure model, also tried competing with other proposed techniques [14]. The image classification without segmenting the images CNN model is used. In this strategy takes theheart cycles into consideration with various starting positions using the Electrocardiogram-signals at the beginning stage. Convolutional Neural Network can produce features with different angles in the stage of testing the data [15]. A lot of information has not been utilized properly which was produced by the clinical practitioners. The introduction of new approaches mitigates the cost also enhance the prediction of CVD in a simple and powerful manner. The different distinctive exploration techniques are taken into consideration in this research for classification and prediction of CVD utilizing Machine Learning and DL algorithms are exceptionally accurate in establishing the viability of these techniques [16].

\section{Overview of Methodology and Results}

Using HRFLM, we come up with a computational strategy with the 3 affiliation regulations of data mining in particular, Predictive, Apriori, and Tertius to discover the variables of CVD on the UCI dataset. The accessible data focuses to the allowance that females having a lesser degree of possibility for CVD contrasted with males. In CVD, exact analysis is essential. Be that as it may, the conventional methodologies are insufficient for precise prediction and analysis. 
HRFLM utilizes Artificial Neural Networks with backpropagation alongside 13 medical features as input. The acquired outcomes are similarly breaking down against customary techniques [17]. The danger levels become high and various credits are utilized for exactness in the conclusion of the illness [18]. The complexity and nature of CVD require an effectual treatment plan. Information mining techniques help in therapeutic circumstances in the clinical field. The information mining strategies are additionally utilized thinking about NN, DT, KNN and SVM. Among a few utilized strategies, the outcomes from SVM end up being valuable in upgrading exactness in the expectation of infection. A module to check heart condition using a non linear methodology is acquainted which recognizes the arrhythmias which means tachycardia, bradycardia, atrial ventricular vacillates, atrial, and numerous others. The presentation viability of this strategy can be assessed from the exactness in the result results dependent on ECG information. ANN preparing is utilized for the exact finding of illness and the forecast of potential anomalies of the particular patient.

Various data mining techniques and prediction strategies, for example, Linear Relapse K-Nearest Neighbours, Neural Network, Support Vector Machines, and Vote have been somewhat mainstream recently to distinguish and anticipate CVD. The tale strategy Vote related to a mixture approach utilizing Naïve Bayes and Linear Regression are used in this research. The dataset gathered from UCI is utilized for directing the analyses the proposed strategy, which brought about $87.34 \%$ precision in the forecast of CVD. The PPCA strategy is bring forward for assessment, in view of three informational indexes of Switzerland, Hungarian, and Cleveland in UCI individually. The strategy extricates the vectors along with vector projection and high covariance is utilized for limiting the component measurement. The component determination with limiting measurement is given to an outspread premise work, which upholds SVM kernal. The aftereffects of the techniques are $85.820 \%, 91.310 \%$, and $82.186 \%$ of UCI informational indexes of Hungarian, Switzerland, and Cleveland individually [19]. The half and half strategy joining MARS, LR, and ANN is presented with unpleasant set methods and the fundamental novel contribution in this research paper. The technique which brought forward is viably decreased the arrangement of basic credits. The leftover ascribes are contributions to ANN, therefore. The CVD datasets are utilized to show the viability of the improvement of the crossbreed approach. The CVD expectation with a multi-facet view proposing Neural Networks. This technique utilizes 13 clinical quality highlights as the info and prepared by backpropagation are extremely exact outcomes in recognizing if the patient has CVD.

We likewise present the Apriori calculation with Support Vector Machine and comparison with 9 different classification techniques to anticipate CVD with more precision. The aftereffects of the order strategy have demonstrated a more serious level of precision and execution in the forecast of CVD contrasted with the other existing strategies. The component choice assumes a conspicuous part in the expectation of CVD. ANN with backpropagation is for better forecast of the sickness. The outcomes acquired from the use of ANN are profoundly exact and exceptionally exact. The hereditary calculation with fuzzy Neural Networks also known as RFNN is presented for the finding of CVD.

From the UCI Dataset out of 297.00 record occurrence of patient data, altogether, are taken for experimentation out of which 250 records are separated for training purpose and the leftover for testing purposes considered. The outcomes have been situated to be fulfilling dependent on the appraisal. A CVD forecast with Support Vector Machines and ANN model is proposed in this research work. In this methodology, two techniques are utilized for the reason of the precision and testing time consumption. The proposed experimentation model organizes the clustered dataset into two separate classes in Support Vector Machine just as Artificial Neural Networks for additional investigation as demonstrated in [20]. The backpropagation of Neural Network with order technique is presented, where HGS is created and afterward, from there on the specific quality arrangement. The exhibition of the BPNN with the classification strategies has been estimated in the preparation stage just like testing stage with the different types of samples. The precision of this method has enhanced the uniformity of total number of available records.

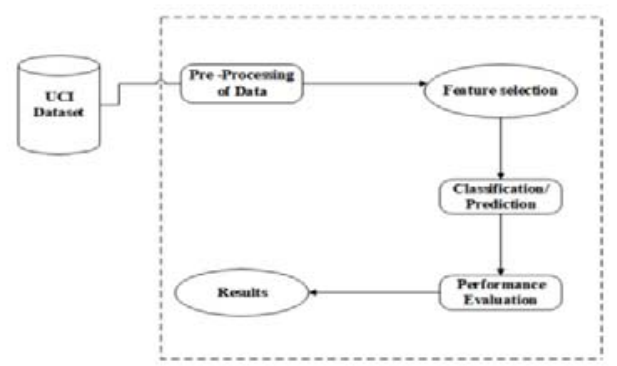

Figure 1. Work flow chart

Table 1: UCI Dataset range and type

\begin{tabular}{|l|l|}
\hline AGE & Numeric $[29$ to $77 ;$ unique $=41 ;$ mean $=54.4 ;$ median $=56$ ] \\
\hline SEX & Numeric $[0$ to $1 ;$ unique $=2 ;$ mean $=0.68 ;$ median $=1]$ \\
\hline CP & Numeric $[1$ to $4 ;$ unique $=4 ;$ mean $=3.16 ;$ median $=3$ ] \\
\hline TESTBPS & Numeric $[94$ to $200 ;$ unique $=50 ;$ mean $=131.69 ;$ median $=130]$ \\
\hline CHOL & Numeric $[126$ to $564 ;$ unique $=152 ;$ mean $=246.69 ;$ median $=241]$ \\
\hline FBS & Numeric $[0$ to $1 ;$ unique $=2 ;$ mean $=0.15 ;$ median $=0$ ] \\
\hline RESTECG & Numeric $[0$ to $2 ;$ unique $=3 ;$ mean $=0.99 ;$ median $=1]$ \\
\hline THALACH & Numeric $[71$ to $202 ;$ unique $=91 ;$ mean $=149.61 ;$ median $=153]$ \\
\hline EXANG & Numeric $[0$ to $1 ;$ unique $=2 ;$ mean $=0.33 ;$ median $=0.00]$ \\
\hline OLPEAK & Numeric $[0$ to $6.20 ;$ unique $=40 ;$ mean $=1.04 ;$ median $=0.80]$ \\
\hline SLOPE & Numeric $[1$ to $3 ;$ unique $=3 ;$ mean $=1.60 ;$ median $=2]$ \\
\hline CA & Categorical $[5$ levels] \\
\hline THAL & Categorical $[4$ levels] \\
\hline TARGET & Numeric $[0.00$ to $4.00 ;$ unique $=5 ;$ mean $=0.94 ;$ median $=0.00]$ \\
\hline
\end{tabular}




\section{Methodology}

In this investigation, we have utilized and Python Jupyter Notebook platform to perform the classification of CVD using the UCI(Cleveland) dataset. Using the visualization libraries whichprovidesa beter perspective and visualization of data, , building the prescient examination, and working climate. Machine Learning measure begins from the data pre-processing stage followed by determination of features based on Decision Tree entropy, modelling performance assessment using classification, and the outcomes with improved exactness. The element choice and demonstrating continue rehashing for different blends of qualities. Table 1 shows the information type and scope of qualities. The exhibition of each method produced dependent on 13 highlights and Machine Learning procedures utilized for every emphasis and execution are recorded. Section 4.1 sums up the information prepreparing, Section 4.2 talks about the component choice utilizing entropy, in Section 4.3 clarifies the characterization with ML methods and Section 4.4 introduced the presentation of the outcomes.

\subsection{Pre-Processing}

CVD information is pre-handled after the assortment of different record types. An aggregate of 303 patient's records contained in the dataset, out of those 6 records have few data values are missing. Those missing records in the data was deleted and the leftover patient's records(297) are utilized for pre-processing. The binary classification and multi-class variable are presented for attributes in the dataset. In order to find out the absence or presence of CVD multi-class variable is utilized. For instance, when the patient has CVD the binary classification value is changed from 0 to 1 , if not the value remains 0 which demonstrates the absence of CVD in patient. The data pre-processing carries out conversion of over clinical values as diagnostic values. Out of 297 patient records demonstrates that 137 records are classified as 1 which resulted in data pre-processing setting up the presence of CVD while the rest of the 160 as 0 which indicates an absence of CVD.

\subsection{Feature Selection}

From the dataset out of 13 , only two clinical attributes concerned to sex and age are used to find out the patient's personal information. The rest of the 11 attributes are examined as crucial clinical information. All these clinical records are very important for learning and diagnosis of the CVD. As explained earlier a lot of machine learning techniques are utilized to find out the CVD namely GLM, NB, DL, LR, RF, DT, SVM, and GBT. The research work was done repeatedly with all machine learning algorithms considering the 13 attributes. In the below figure-2 flowchart shows the HRFLM prediction method.

\subsection{Classification}

The dataset clustering is completed by taking the criteria and variables of Decision Tree features as basis. Later each clustered dataset is fed to the classifiers to measure the performance and the best algorithms are selected using the above outcomes based on very low error rate. Further optimization of performance using the decision tree cluster in which the error rate is high and respected feature extraction using classifiers. The classifier performance is furtherly examined for error mitigation on the opted dataset.

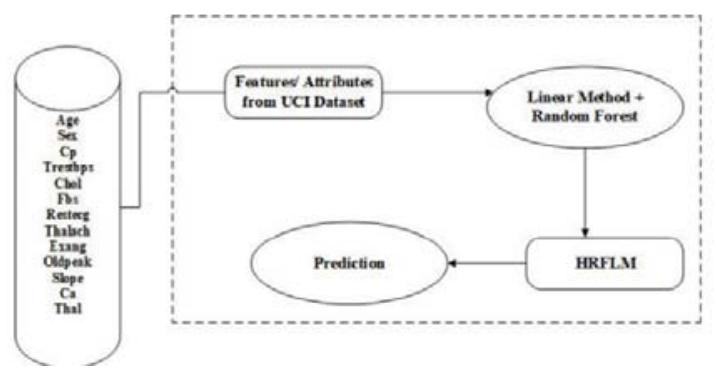

Figure 2. CVD Predictionusing HRFLM

\subsection{Performance Evaluation}

A several basic evaluations of performance metrics like precision, classification error and accuracy was taken into consideration for the performance computation efficiency of this research model. In this current scenario accuracy means the examples at what percentage rate accurately predicting out of all the instances available. The percentage of exact prediction is defined as precision in the true class of examples. The percentage of available errors or accuracy lacking are defined using the error. To distinguish the features of CVD, three performance variables are utilized which helps to understand better about the behaviour of the different blends of selecting the features. Machine Learning algorithms focuses on the model which performs the best contrasted with existing strategies. We are coming up to use HRFLM, which creates highly précised and less error rate classification to predict the CVD. The exhibition of all the classifiersisevaluated separately and outputs aresaved for future assessments.

\subsection{Algorithms}



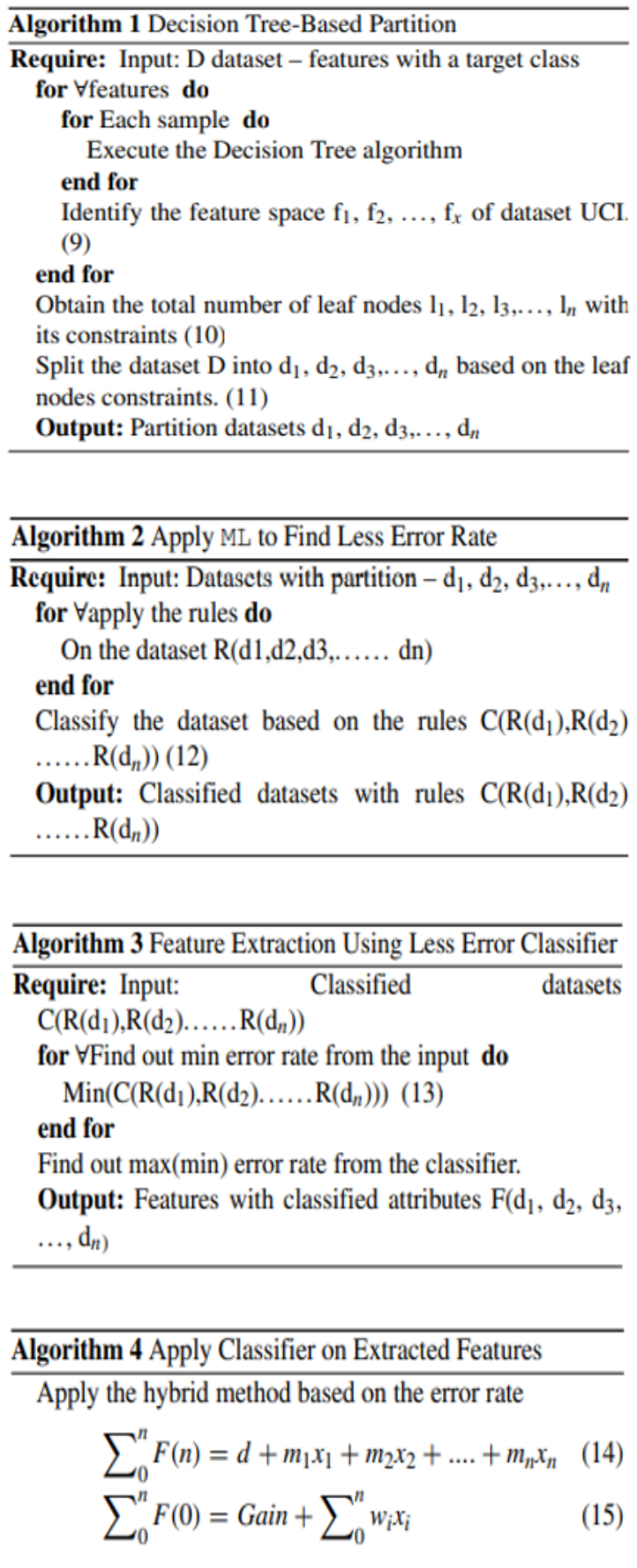

\section{Experimentational Environment}

\subsection{Datasets}

CVD datasets were gathered from UCI AI repository. Further UCI have four different datasets available in their repository namely: Cleveland, the VA Long Beach, Switzerland, and Hungary. The Cleveland data set was chosen for this examination since it is a normally utilized information base for Machine Learning specialists with thorough and full-fledged records. In the repository dataset holds 303.00 patient records. The
Cleveland dataset which is holding 76.00 variables, the informational index gave in the archive outfits data subset of just 14 variables. The below Table 1 portrays the depiction and kind of qualities. Total 13 attributes that include in the forecast of CVD, where just one quality fills in as the yield or the anticipated characteristic to find out the CVD in patient.

The dataset consists various attributes to display the finding of CVD in patients on various measures, which starts 0 and ends at 4 . In this kind of scenario, 0 addresses the shortfall of CVD and every one of the qualities from 1 and ends at 4 addresses patients with CVD, where the measurements alludes to the seriousness of the infection 4 indicates most elevated. In Figure 1 indicates the circulation of the attributes out of the recognized 303 records.
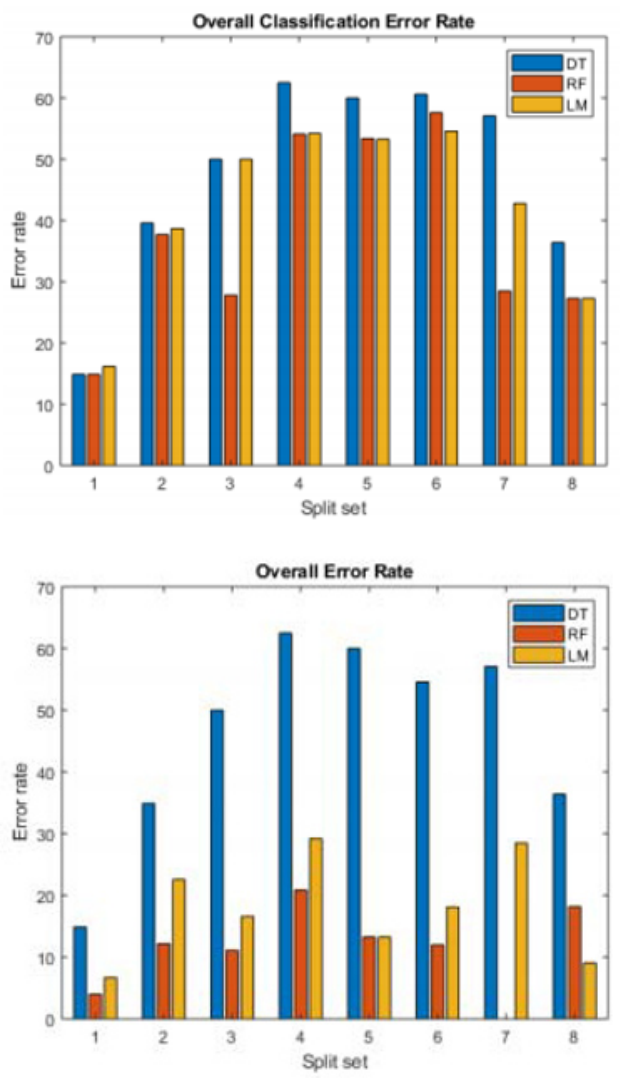

Figure 3.Dataset Over all Error rate classification error rate

\subsection{Experiment interface and Evaluation}

We have utilized anPython Jupyter Notebook to execute the CVD classification using the UCI dataset. Figure-1 portrays the assessment of the investigation step-by-step organizes. In the initial stage, the data is stacked and arranged for pre-processing. There are 13 different attributes (AGE, EXANG, CP, SEX, CHOL, TREEBPS, RESTECG, FBS, OLDPEAK, THALACH, CA, SLOPE, TARGET, and THAL) is chosen among 
the pre-processed dataset of CVD. The 3 different detection models to find CVD are Decision Tree, Random Forest, and are utilized to build up the classification model. The assessment of the classification is done using confusion matrix. Absolutely, four results are created by the disarray framework, in particular FN (False Negative) TN (True Negative), FP (False Positive), and TP (True Positive).

$$
\begin{aligned}
\text { Accuracy } & =(\mathrm{TN}+\mathrm{TP}) /(\mathrm{TN}+\mathrm{TP}+\mathrm{FN}+\mathrm{FP}) \\
& =105+155 / 295=0.8847 \\
\text { Sensitivity } & =(\mathrm{TP} / \mathrm{TP}+\mathrm{FN})=155 / 155+12=92.8 \\
\text { Specificity } & =(\mathrm{TN} / \mathrm{TN}+\mathrm{FP})=105 / 105+22=82.6
\end{aligned}
$$

The accompanying measures are utilized for the estimation of the specificity, accuracy, and sensitivity.

\section{Evaluation Results}

The present CVD prediction system is structured using 13 aspects and calculated the modelling techniques accuracy. The various classification model results are mentioned in Table 2, which portrays and differentiates the accuracy with other classification models along with the comparison of, classification error, Accuracy, Fmeasure, Precision, specificity, and sensitivity. The most elevated accuracy is accomplished by the proposed classification method (HRFLM) algorithmic model is

\begin{tabular}{|c|c|c|c|c|c|c|}
\hline Models & Accuracy & $\begin{array}{c}\text { Classification } \\
\text { error }\end{array}$ & Precision & $\begin{array}{c}\mathrm{F}- \\
\text { measure }\end{array}$ & Sensitivity & Specificity \\
\hline Naire Bayes & 75.8 & 24.2 & 90.5 & 84.5 & 79.8 & 60.0 \\
\hline Genteralized Linear Mocel & 85.1 & 14.9 & 88.8 & 91.6 & 94.9 & 20.0 \\
\hline Logistic Regression & 82.9 & 17.1 & 89.6 & 90.2 & 91.1 & 25.0 \\
\hline Deep Leaming & 87.4 & 12.6 & 90.7 & 92.6 & 95 & 33.3 \\
\hline Decision Treet & 85 & 15.0 & 86 & 91.8 & 98.8 & 0.0 \\
\hline Random Forest & 86.1 & 13.9 & 87.1 & 92.4 & 98.8 & 10.0 \\
\hline Gradient Boosted Trees & 78.3 & 21.7 & 94.1 & 86.8 & 80.7 & 60.0 \\
\hline Support Vector Machine & 86.1 & 13.9 & 86.1 & 92.5 & 100 & 0.0 \\
\hline VOTE & 87.41 & 12.59 & 90.2 & 84.4 & . & . \\
\hline HRFLM & 88.4 & 11.6 & 90.1 & 90 & 92.8 & 82.6 \\
\hline
\end{tabular}
compared with other algorithms.

Table 2: Results of differentalgorithmsalongwithproposed model

Table 3: Results of different classification models and

\begin{tabular}{|c|c|c|c|c|c|c|}
\hline & \multicolumn{2}{|c|}{$\begin{array}{l}\text { Overall } \\
\text { error } \\
\text { rate }\end{array}$} & \multirow[t]{2}{*}{$\begin{array}{l}\text { Best } \\
\text { Model }\end{array}$} & \multicolumn{2}{|c|}{$\begin{array}{l}\text { Overall clas- } \\
\text { sification er- } \\
\text { ror rate }\end{array}$} & \multirow[t]{2}{*}{$\begin{array}{l}\text { Best } \\
\text { Model }\end{array}$} \\
\hline $\begin{array}{l}\text { Data } \\
\text { Split }\end{array}$ & RF & LM & & RF & LM & \\
\hline 1 & 4 & 6.7 & RF & 14.9 & 16.2 & $\begin{array}{l}\text { DT } \\
\text { /RF }\end{array}$ \\
\hline 2 & 12.2 & 22.6 & RF & 37.7 & 38.7 & RF \\
\hline 3 & 11.1 & 16.6 & RF & 27.8 & 50 & RF \\
\hline 4 & 20.9 & 29.2 & RF & 54.1 & 54.2 & $\mathrm{RF}$ \\
\hline 5 & 13.3 & 13.3 & $\begin{array}{l}\text { RF/ } \\
\text { LM }\end{array}$ & 53.4 & 53.3 & LM \\
\hline 6 & 12 & 18.1 & RF & 57.6 & 54.6 & LM \\
\hline 7 & 0 & 28.5 & RF & 28.5 & 42.8 & RF \\
\hline 8 & 18.2 & 9.1 & LM & 27.3 & 27.3 & $\begin{array}{l}\text { RF/ } \\
\text { LM }\end{array}$ \\
\hline
\end{tabular}
proposed model.

\begin{tabular}{|l|l|l|l|l|l|l|l|l|}
\hline & \multicolumn{9}{|c|}{ Overall error rate } & \multicolumn{2}{l|}{$\begin{array}{l}\text { Best } \\
\text { Model }\end{array}$} & \multicolumn{3}{|l|}{ Overall classification error rate } & $\begin{array}{l}\text { Best } \\
\text { Model }\end{array}$ \\
\hline $\begin{array}{l}\text { Data } \\
\text { Split }\end{array}$ & DT & RF & LM & & DT & RF & LM & \\
\hline 1 & 14.9 & 4 & 6.7 & RF & 14.9 & 14.9 & 16.2 & $\begin{array}{l}\text { DT } \\
\text { /RF }\end{array}$ \\
\hline 2 & 34.9 & 12.2 & 22.6 & RF & 39.6 & 37.7 & 38.7 & RF \\
\hline 3 & 50 & 11.1 & 16.6 & RF & 50 & 27.8 & 50 & RF \\
\hline 4 & 62.5 & 20.9 & 29.2 & RF & 62.5 & 54.1 & 54.2 & RF \\
\hline 5 & 60 & 13.3 & 13.3 & $\begin{array}{l}\text { RF/ } \\
\text { LM }\end{array}$ & 60 & 53.4 & 53.3 & LM \\
\hline 6 & 54.6 & 12 & 18.1 & RF & 60.6 & 57.6 & 54.6 & LM \\
\hline 7 & 57.1 & 0 & 28.5 & RF & 57.1 & 28.5 & 42.8 & RF \\
\hline 8 & 36.4 & 18.2 & 9.1 & LM & 36.4 & 27.3 & 27.3 & $\begin{array}{l}\text { RF/ } \\
\text { LM }\end{array}$ \\
\hline
\end{tabular}

Table 4: HRFLM Results

\section{Conclusion}

Recognizing the preparation of raw medical datasets of CVD will be useful in the future to save the life of human and also early detection of cardio vascular problems. Artificial Intelligence methods used in this research to so process the raw data to make sense and train the system to get to know the attributes of CVD. The CVD prediction is difficult also plays a crucial part in the medical science industries. Nonetheless, the death rate could be definitely controlled in case of the effect of sickness is identified at the starting stages and deterrent measures are received as quickly as time permits. Further augmentation of this experiment is profoundly attractive to take care of the assessments to real time datasets instead of basically speculative techniques and recreations. The proposed methodology called HRFLM approach is experimented to consolidate the variables of Linear Method (LM) and Random Forest (RF). HRFLM is proved up that the model is very accurate in the forecasting of CVD. The future enhancements of this model will be experimented using various other combinations of $\mathrm{AI} / \mathrm{ML} / \mathrm{DL}$ algorithms. Moreover, new element determination approaches would be created in order to get highly accurate perception of the crucial data to enhance the performance of CVD prediction.

\section{References}

[1] M. Durairaj and V. Revathi, International Journal of Scientific \& Technology (2015)

[2] V. Gadepally, T. Mattson, M. Stonebraker, F. Wang, G. Luo, Y. Laing, and A. Dubovitskaya, Heterogeneous Data Management, Polystores, and Analytics for Healthcare: VLDB 2019 Workshops, Poly and DMAH, Los Angeles, CA, USA, August 30, 2019, Revised Selected Papers (Springer Nature, 2019)

[3] B. S. S. Rathnayakc and G. U. Ganegoda, in 2018 3rd International Conference for Convergence in Technology (I2CT), pp. 1-6(2018)

[4] M. Aslam and J. K, in 2021 International Conference on Computer Communication and Informatics (ICCCI) (2021)

[5] J. P. Kelwade and S. S. Salankar, in 2016 IEEE First International Conference on Control, 
Measurement and Instrumentation (CMI) (2016)

[6] A. H. Alkeshuosh, M. Z. Moghadam, I. A. Mansoori, and M. Abdar, in 2017 International Conference on Computer and Applications (ICCA) (2017)

[7] F. Dammak, L. Baccour, and A. M. Alimi, in 2015 IEEE International Conference on Fuzzy Systems (FUZZ-IEEE) (2015)

[8] M. J. Liberatore and R. L. Nydick, Eur. J. Oper. Res. 189, 194 (2008)

[9] Annual IEEE/IFIP International Conference on Dependable Systems and Networks, 48th Annual IEEE/IFIP International Conference on Dependable Systems and Networks: DSN 2018 : Proceedings : 25-28 June 2018, Luxembourg City, Luxembourg (IEEE, 2018)

[10] J. Jose, J. S. Vimali, P. Ajitha, S. Gowri, A. Sivasangari, and B. Jinila, in 2021 th International Conference on Trends in Electronics and Informatics (ICOEI)

(2021)

K. Prasanna Lakshmi and C. R. K. Reddy, in 2010

[11] International Conference on Networking and Information Technology (2010)

[12] J. Wu, S. Luo, S. Wang, and H. Wang, IEEE Internet of Things Journal 6, 2463 (2019)

[13] J. Wu, K. Ota, M. Dong, and C. Li, IEEE Access 4, 416 (2016)

[14] "R. Das, I. Turkoglu, and A. Sengur, Expert Syst. Appl. 36, 7675 (2009)

[15] IEEE Staff, 2019 41st Annual International Conference of the IEEE Engineering in Medicine and Biology Society (EMBC) (IEEE, 2019)

[16] J. Nahar, T. Imam, K. S. Tickle, and Y.-P. P. Chen, Expert Syst. Appl. 40, 1086 (2013)

[17] D. K. Ravish, K. J. Shanthi, N. R. Shenoy, and S. Nisargh, in 2014 International Conference on Contemporary Computing and Informatics (IC3I) (2014)

[18] C. U. Kumari, S. J. Prasad, and G. Mounika, (2019)

[19] T. Mahboob, R. Irfan, and B. Ghaffar, in 2017 Internet Technologies and Applications (ITA) (2017)

[20] O. Goldman, O. Raphaeli, E. Goldman, and M. Leshno, Qual. Manag. Health Care (2021)

[21] M. S. Amin, Y. K. Chiam, and K. D. Varathan, Telematics and Informatics 36, 82 (2019)

S. C. Satapathy, K. Srujan Raju, J. K. Mandal, and

[22] V. Bhateja, Proceedings of the Second International Conference on Computer and Communication Technologies: IC3T 2015, Volume 1 (Springer, 2015)

[23] B. Dhanalaxmi, G. A. Naidu, and K. Anuradha, Procedia Comput. Sci. 46, 432 (2015) 